\title{
Monte Carlo calculation of the spatial response (Modulated Transfer Function) of a scintillation flat panel and comparison with experimental results
}

\author{
Belén Juste $^{1 *}$, Rafael Miró ${ }^{1}$, Paula Monasor ${ }^{1}$ and Gumersindo Verdú ${ }^{1}$ \\ ${ }^{1}$ Instituto de Seguridad Industrial, Radiofísica y Medioambiental (ISIRYM). \\ Universitat Politècnica de València. Camí de Vera s/n. 46022. Valencia, Spain. \\ *Corresponding author: bejusvi@iqn.upv.es 0034-963879631
}

Keywords: X-ray imaging; Modulation transfer function (MTF); Slit method; Monte Carlo simulation; MCNP6, flat panel scintillator

\begin{abstract}
Phosphor screens are commonly used in many X-ray imaging applications. The design and optimization of these detectors can be achieved using Monte Carlo codes to simulate radiation transport in scintillation materials and to improve the spatial response.

This work presents an exhaustive procedure to measure the spatial resolution of a scintillation flat panel image and to evaluate the agreement with data obtained by simulation. To evaluate the spatial response we have used the Modulated Transfer Function (MTF) parameter. According to this, we have obtained the Line Spread Function (LSF) of the system since the Fourier Transform (FT) of the LSF gives the MTF.

The experimental images were carried out using a medical X-ray tube (Toshiba E7299X) and a flat panel (Hammamatsu C9312SK). Measurements were based on the slit methodology experimental implementation, which measures the response of the system to a line. LSF measurements have been performed using a $0.2 \mathrm{~mm}$ wide lead slit superimposed over the flat panel.

The detector screen was modelled with MCNP (version 6) Monte Carlo simulation code in order to analyze the effect of the acquisition setup configuration and to compare the response of scintillator screens with the experimental results. MCNP6 offers the possibility of studying the optical physics parameters (optical scattering and absorption coefficients) that occur in the phosphor screen.

The study has been tested for different X-ray tube voltages, from 100 to $140 \mathrm{kV}$. An acceptable convergence between the MTF results obtained with MCNP6 and the experimental measurements have been obtained.
\end{abstract}

\section{Introduction}

Digital X-ray imaging detectors based on scintillating screens are used for general radiography, mammography, dental and non-destructive testing (NDT) applications.

The design and optimization of these detectors can be achieved using experimental methods, which involve expensive and laborious trials and errors. A potential option lies in using Monte Carlo code to simulate radiation transport in scintillation screens in order to improve the spatial response. 
To validate the simulated results, we have compared them with experimental measurements. Since the specifications of the methods employed in the experiments have a considerable impact on the precision of the results, a careful analysis of the experimental procedure is required.

The Modulation Transfer Function is widely recognized as the most relevant metric of resolution in radiographic imaging. The most commonly used methodologies for determining the spatial response of scintillation screens are the slit method and the edge method [1]. In the edge method, the Edge Spread Function (ESF) is derived in order to obtain the Line Spread Function (LSF) of the system and the Fourier Transform (FT) of the LSF gives the MTF. Taking the derivative of the ESF, which usually increases the input noise, may be avoided by using the slit method, which also reduces artifacts due to scattering in the detector. This method measures the response of the system to a line (taking into account the slit width). The MTF is calculated by taking the FT of the LSF.

Monte Carlo simulation is an accurate tool to analyze the physics transport and interactions inside the scintillator screens, since the physics processes or geometry of the screen do not need simplifying assumptions.

\section{Experimental measures}

The slit methodology has been used for measuring the LSF, which involves taking the response of the system to a line.

LSF measurements have been carried out using a $0.2 \mathrm{~mm}$ wide lead slit (1 mm thick) superimposed on a commercial scintillator, Hammamatsu flat panel (Hammamatsu C9312SK) (Fig.1) [2], [3].

\begin{tabular}{|c|c|c|}
\hline Slice & Material & Thickness [mm] \\
\hline Scintillator & $\mathrm{Gd}_{2} \mathrm{O}_{2} \mathrm{~S}$ & 0.14 \\
Fibreoptic & $\mathrm{Al}_{2} \mathrm{O}_{3}$ & 3.0 \\
Semiconductor & $\mathrm{Si}$ & 1.5 \\
Foil & $\mathrm{Cu}$ & 0.4 \\
Absorptive layer & $\mathrm{Pb}$ & 1.5 \\
\hline
\end{tabular}

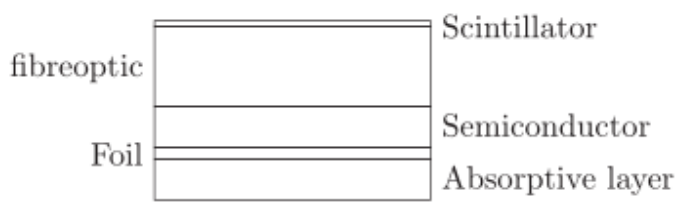

Figure 1. Internal structure of the Hammamatsu C9312SK flat panel, gadolinium oxysulfide $\left(\mathrm{Gd}_{2} \mathrm{O}_{2} \mathrm{~S}\right)$ powder phosphor screen.

The slit scintillator system was irradiated using a W-target medical X-ray tube (Toshiba E7299X), at 100, 120 and $140 \mathrm{kV}$ tube voltages.

The x-ray beam was collimated in order to irradiate the complete flat panel screen $(124.8 \times 115.2$ $\mathrm{mm}$ ). The flat panel HAMMAMATSU C9312SK has a pixel size of $50 \mu \mathrm{m} \times 50 \mu \mathrm{m}$ and $140 \mu \mathrm{m}$ thick gadolinium oxysulfide scintillator. It consist on a $1024 \times 1024$ square pixels matrix of 14 bits. 
The process is carried out for the tube voltages of 100, 120 and $140 \mathrm{kV}$. Figure 2 shows the image corresponding to a $140 \mathrm{kV}$ X-Ray beam and its corresponding LSF curve.
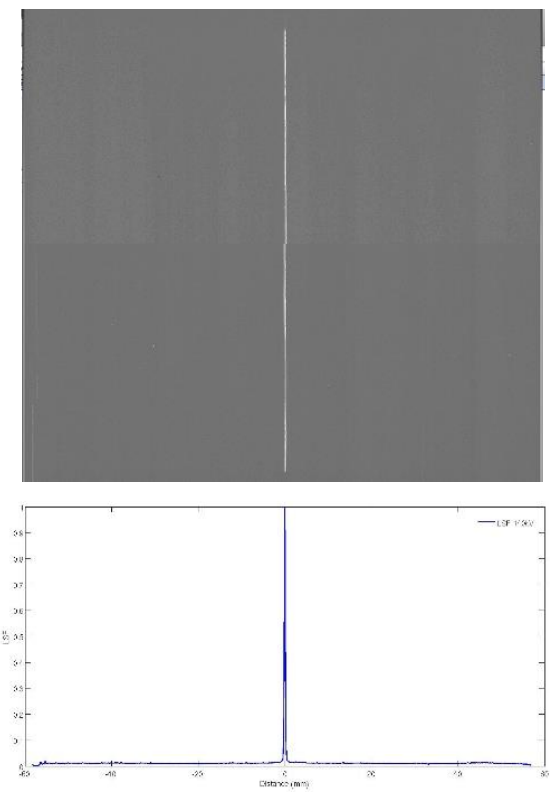

Figure 2. $140 \mathrm{kV}$ x ray Flat panel image and its associated LSF.

Figure 3 shows the LSF curves obtained using 100, 120 and $140 \mathrm{kV}$ tube setting.

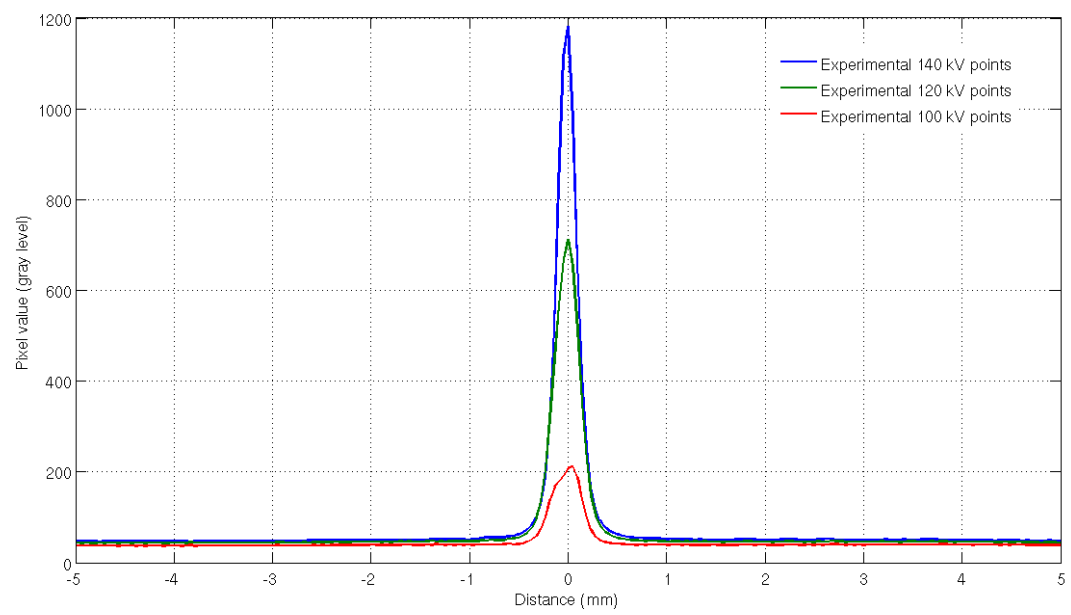

Figure 3. 100, 120, $140 \mathrm{kV}$ image LSF.

\section{MCNP6 simulations}

In the present work, the response of scintillator screens is simulated with the Monte Carlo code MCNP6 [4] in the case of a beam of X-ray photons orthogonally impinging onto the slit and scintillation screen.

An ideal detector situated behind the screen records all the impacts of the optical photons coming out of the phosphor. In order to compare with the experimental results, the complete structure of the Lanex Fine scintillator screen has been simulated (see Fig. 1). The optical system (Pb glass, 
CCD camera) was not included in the simulations. Moreover, to reduce the complexity of the simulation, the microstructure of the powder phosphor is not described explicitly. Instead of that, the phosphor is considered as a homogeneous medium. Figure 4 shows a sketch of the modelled geometry.

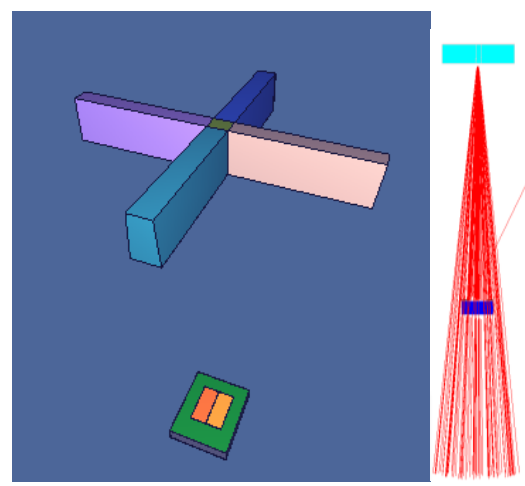

Figure 4. X-Ray tube, collimator and flat panel modeled with MCNP6

The x-ray spectrum has been simulated according to the catalog IPEM Report 78, "Catalogue of Diagnostic X-Ray Spectra \& Other Date" [5], which gives the spectrum data for a tungsten target, starting with semi-empirical calculations, for voltages from 30 to $140 \mathrm{kV}$.
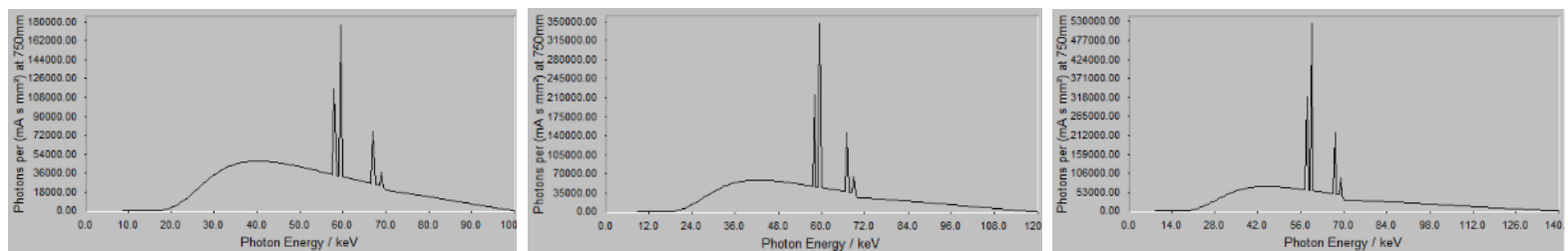

Figure 5. IPEM Report 78 X ray spectrum: 100, 120 and 140 kV.

HVL Alumninium $2 \mathrm{~mm}$ filtration was obtained via Piranha detector measures [6]. This solidstate detector was placed just on the top of the flat panel screen to obtain the HVL, which is calculated by using the measured $\mathrm{kV}$ values, Figure 6.

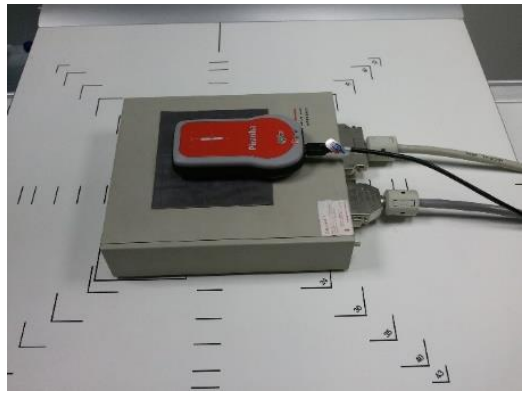

Figure 6. Dose and HVL measures using Piranha detector. 
One of the main new capabilities on the MCNP version 6 is the extension of photon and electron transport to lower energies.

It is now possible to transport photons and electrons below $1 \mathrm{keV}$. We have set the new lower limits for energy cutoffs, which are $1 \mathrm{eV}$ for photons and $10 \mathrm{eV}$ for electrons.

The simulated processes that have taken into account in this MCNP6 model are photoelectric effect, Compton and Rayleigh scattering for the X-ray photons, pair production, multiple scattering, ionization and bremsstrahlung for the electrons, atomic de-excitation by fluorescence and Auger effect after photoelectric effect and optical absorption and scattering for the visible photons.

The tally used to estimate flat panel detector image has been the FMESH4 tally, associated with its respective flux-to-dose conversion factors, which registers the pulse-height distribution modified to energy units at the bottom surface of the scintillator. This tally has registered just photons between 1 and $10 \mathrm{eV}$, to concentrate only optical photons emerging from GOS material.

Since low energy particles transport increases calculation time, the simulation has included a phase space surface over the scintillator layer of the detector. First simulation did not include optical transport. It was activated on the second part of the simulation where phase space was used as source and only the transport in the scintillator was considered. Each simulation run follows the transport of 100 million histories particles, in order to obtain a statistical error always below the $5 \%$.

Figure 7 shows the resulting image obtained registering the optical photons with MCNP6 in order of taking the response of the system to a line for a $140 \mathrm{kV}$ setting tube.

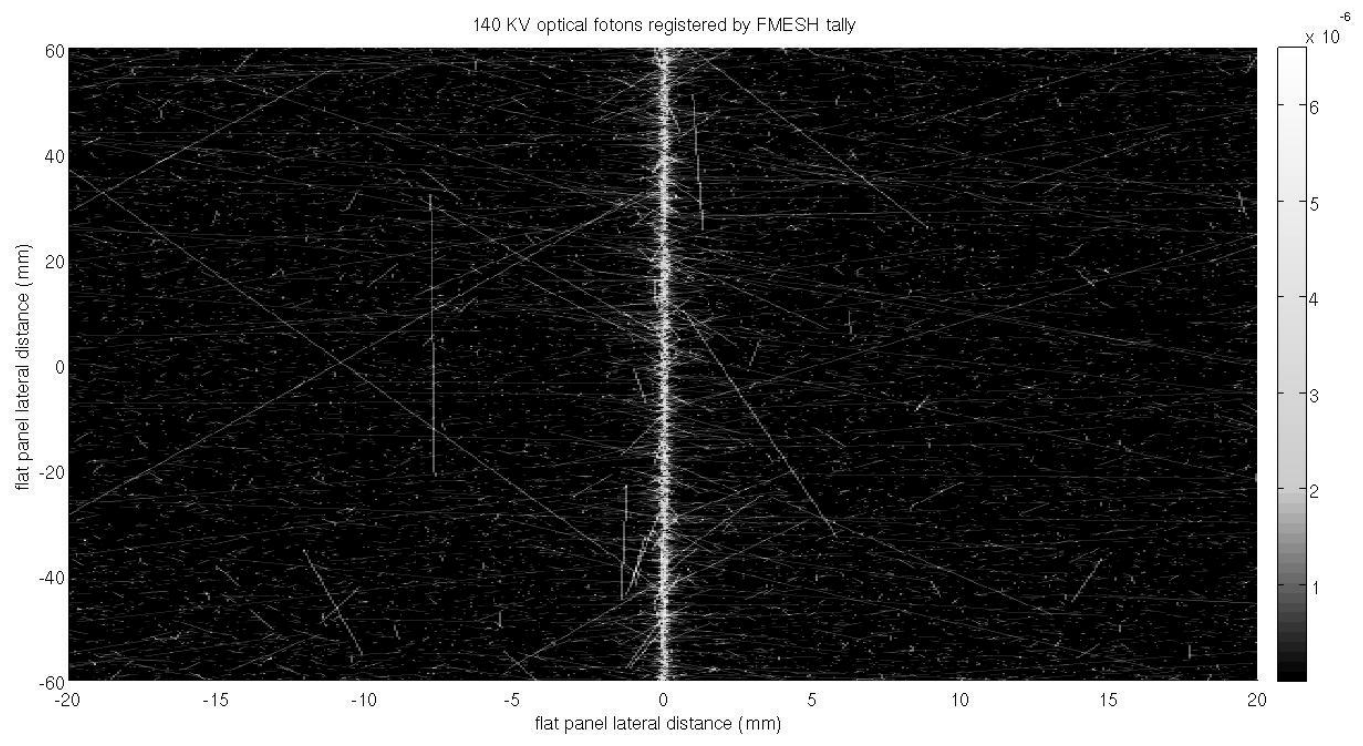

Figure 7. Flat panel image obtained using MCNP6, $140 \mathrm{kV}$ beam. 
Figure 8 displays the comparison of LSF curve obtained via simulation and experimentally, for 100,120 and $140 \mathrm{kV}$ X-ray.
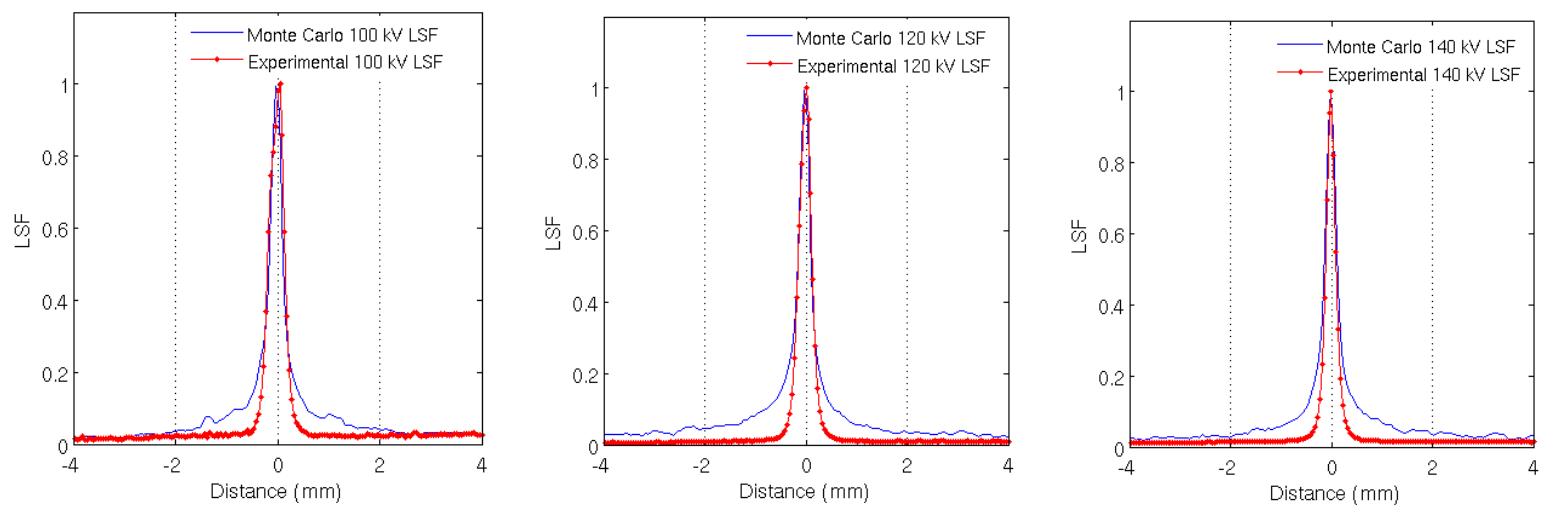

Figure 8. LSF for 100, 120 and $140 \mathrm{kV}$

We can see in figure 8 that the shape of the central peak in the simulated LSFS is in satisfactory agreement with the experimental data. However, the experimental tails of the LSFS are significantly below the simulated results.

A possible cause of this discrepancy may be the influence of the phosphor microstructure, which is not explicitly taken into account in the simulation model. Moreover, the optical processes at the interface between two media (reflection, refraction) are not considered in this version of MCNP6, although it will be included in next future.

Finally, after calculating the Fourier Transform of LSF curves, a direct MTF comparison between the simulated and measured results is studied. Figure 9 shows the comparison of MTF parameter of simulation results and experimental images.
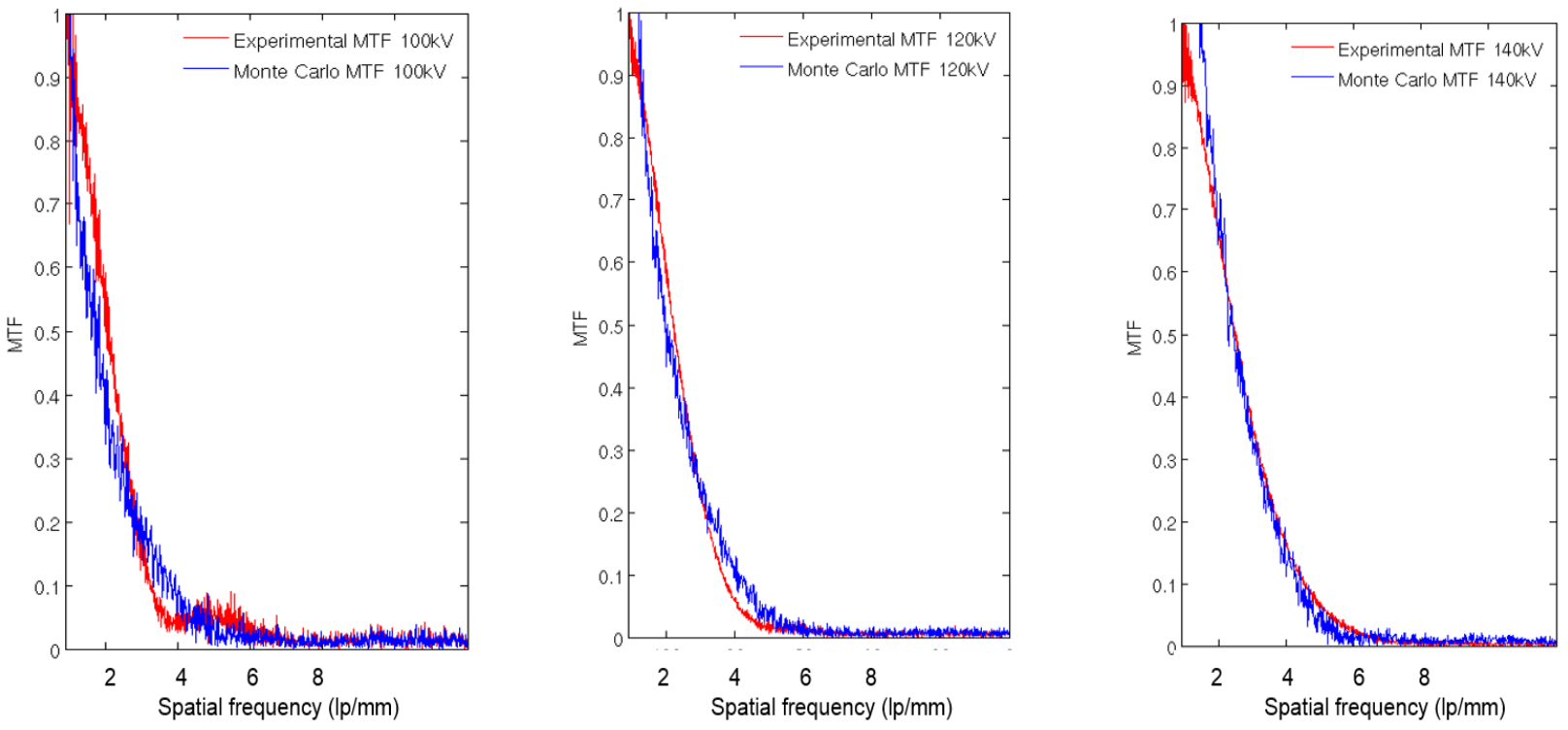
Figure 8. MTF curves for 100, 120 and $140 \mathrm{kV}$.

For an ideal system that does not modificates the entry, the MTF information would be 1 for all spatial frequencies. For non-ideal detectors the MTF will decrease as it increases the spatial frequency, i.e. is less contrast in the image as it decreases the size of the input objects. While closest to 1 is the value of the MTF at high frequencies, the better the spatial resolution of the system.

A criterion to determine the spatial resolution of a system consists of measuring the MTF of the system and then identificate which spatial frequency corresponds to 0.1 the MTF. Spatial resolution $($ length $)=1 /(2 *$ Spatial frequency $)$.

In this case, as figure 8 represents, the spatial resolution is $0.125 \mathrm{~mm}$, and maximum differences between simulated and experimental values are below $5 \%$.

\section{Conclusions}

The MCNP6 model allows studying the spatial response of the scintillation screens; therefore, it can be used as an interesting tool in technological developments of scintillator screens, since it allows studying new configurations and geometries and improving the imaging performance. The model can be applied with a small number of adjustments (related to the screen structure) to any geometry of scintillator screens.

Monte Carlo simulation are the most suitable approach for the simulation of scintillation screens, in comparison with analytical models or deterministic approaches.

\section{Acknowledgements}

This work was partially supported through "Programa para la innovación e incentivación" of the Polytechnic University of Valencia "INNOVA 2012". This work has been done under the project: Monte Carlo Treatment Planning System: Software for the high precision dosimetry calculations in radiotherapy (SP20120824).

\section{References}

[1] Jerrold T. Bushberg, John M. Boone. The Essential Physics of Medical Imaging. ISBN-13: 9780781780575 Edition: Third, North American Edition.

[2] HAMAMATSU PHOTONICS K.K., Solid State Division. www.hamamatsu.com

[3] N Reims, F Sukowski and N Uhlmann, Modelling of scintillator based flat-panel detectors with Monte Carlo simulations, 12th International Workshop on Radiation Imaging Detectors (IWORID) Vol. 6, 2011.

[4] Los Alamos Laboratory, Quick-Start Guide to Low-Energy Photon/Electron Transport in MCNP6, Hughes, H. Grady II, LA-UR-12-21068, 2013.

[5] Catalogue of Diagnostic X-Ray Spectra \& Other Date. Report 78. ISBN, 090418188 X. Copyright IPEM.

[6] Piranha, RTI, Sweden. http://www.rti.se 